\title{
LLR Computation for Multistage Decoding
}

\author{
Abir Ben Hadj Fredj \\ Telecom ParisTech \\ Paris, France \\ abir.benhadjfredj@telecom-paristech.fr
}

\author{
Jean-Claude Belfiore \\ Telecom ParisTech \\ Paris, France \\ jean-claude.belfiore@telecom-paristech.fr
}

\begin{abstract}
Due to their linear and highly symmetrical structure, lattices are becoming of a great interest as potential transmission schemes. Lattice codes suggest a common view of channel and source coding and new tools for the analysis of information network problems. Several constructions have been proposed to build these lattices, some of which are based on multi-level coding and multistage decoding such as constructions $D$ and $\pi_{D}$. Soft-decision decoders corresponding to the different nested error-correcting codes used to construct such lattices need at each stage the computation of a soft input, namely LogLikelihood Ratios. In this paper, we give an efficient computation of LLRs based on Jacobi theta functions for three different types of constructions; Binary construction D, Quaternary construction $D$ and Binary construction $\pi_{D}$.
\end{abstract}

\section{INTRODUCTION}

In Gaussian multiple access channels (MAC), where two or more users transmit information to the same receiver over a gaussian channel, interference has always been considered as nuisance. Several methods have been developed to avoid interference in MAC such as orthogonal access to the channel techniques (FDMA,TDMA..). However, avoiding interference may impact data rates as they decrease when the network size increases. Physical-layer network coding introduced in 2006 by Zhang et al. in [1], comes up with a revolutional concept allowing to harness interference instead of avoiding it. Based on this new concept, B. Nazer and M. Gastpar in [2], proposed a new strategy, Compute and Forward, that enables relays to decode linear equations of the transmitted messages using the noisy linear combinations provided by the channel. In order to be decoded reliably, the messages must be lattice points whose algebraic structure enables that integer linear combinations of these points remain a lattice point. Several binary and M-ary lattice constructions have been proposed to construct lattices such as construction $A$ and other equivalent constructions namely $B, D, \pi_{A}$ and $\pi_{D}$. One of the main drawbacks of construction $A$ is that in order to be good for AWGN channel, the underlying linear codes have to be implemented over very large prime fields which in turn result in high decoding complexity. Constructions $D$ and $\pi_{D}$ which are based on multi-level coding guarantee a lowcomplexity encoding scheme as well as a practical decoding based on multistage decoding. Different error-correcting codes are used in these lattice constructions. For construction $D$ and for construction $\pi_{D}$, one must have respectively a family of nested codes and a family of equivalent codes. At the receiver side, in order to decode one lattice point, we must decode all the codewords corresponding to the different used errorcorrecting codes. In the binary case, Log-Likelihood Ratios (LLRs) which are the inputs for the soft-decoding algorithms must be calculated at each stage of the multistage lattice decoder.

$$
\operatorname{LLR}=\log \frac{\mathbb{P}\left(y_{i j}^{\prime} \mid x_{i j}=0\right)}{\mathbb{P}\left(y_{i j}^{\prime} \mid x_{i j}=1\right)}
$$

where $y_{i j}^{\prime}$ and $x_{i j}$ are respectively the $j^{\text {th }}$ received bit after the modulo operation and the $j^{\text {th }}$ transmitted bit at the $i^{\text {th }}$ level of the multistage decoder. For Compute and Forward, decoding is performed over the whole lattice, therefore LLRs are computed over the whole lattice which induce an infinite sum of exponentials. Thus, the computations of exact LLRs are very complex for a practical implementation.

In this paper, we propose an LLR computation for gaussian channels based on Jacobi theta functions. To calculate LLRs, we only need to know the value of two types of theta functions. These values can be precalculated easily and stored in lookup tables.

This paper is organized as follows; In the second section, we give some basic definitions on lattices and different constructions as well as the system model. We will also provide some fundamental computation rules of theta functions that we will exploit to perform our calculations. In section three, we will deal with the Gaussian channels and give our LLR expressions as functions of Jacobi theta functions for three different lattice constructions.

Throughout this paper, vectors and matrices are denoted by lowercase and uppercase bold letters, such as $\mathbf{a}$ and $\mathbf{A}$, respectively. The probability of a given event $E$ is denoted by $\mathbb{P}(E)$.

\section{Preliminaries AND SyStem MODEl}

An $n$-dimensional euclidean lattice $\Lambda$ is an additive discrete subgroup of $\mathbb{R}^{n}$. Roughly speaking, a lattice is a periodic arrangement of points in the $n$-dimensional Euclidean space [3]. The fundamental Voronoi region, $R_{\Lambda}$, of a lattice $\Lambda$, is the set of all points in $\mathbb{R}^{n}$ that are closest to the zero vector. A pair of euclidean lattices $\left(\Lambda_{1}, \Lambda_{2}\right)$ is nested if $\Lambda_{2} \subseteq \Lambda_{1}$, and the finite quotient group $\Lambda_{1} / \Lambda_{2}$ is called a lattice partition whith index $\left|\Lambda_{1} / \Lambda_{2}\right|$. The partition index is the number of cosets of $\Lambda_{2}$ in $\Lambda_{1}$. A family of $r$ nested lattices defines a lattice 
partition chain $\Lambda_{1} / \Lambda_{2} / \ldots . . / \Lambda_{r}$.

Multi-level coding is a practical encoding scheme for lattices. It uses several error-correcting codes and the transmitted symbols are constructed by combining symbols of codewords of these codes [4]. It has been proved in [5] that multi-level coding (MLC) and multistage decoding (MSD)[6] suffice to approach capacity if the rates at each level are appropriately chosen. Construction $D$ is based on this multi-level nature.

As an example, let us consider the lattice partition chain $\mathbb{Z}^{n} / 2 \mathbb{Z}^{n} / 4 \mathbb{Z}^{n} / \ldots .2^{r} \mathbb{Z}^{n}$ and the linear binary code $C_{i}\left(n, k_{i}\right)$, the code associated to the partition $2^{i} \mathbb{Z}^{n} / 2^{i+1} \mathbb{Z}^{n}$. We consider as well a family of $\mathrm{r}$ nested codes $C_{0} \subseteq C_{1} \subseteq \ldots . \subseteq C_{r-1}$. Then the lattice $\Lambda$ can be defined as follows:

$$
\Lambda_{1}=2^{r} \mathbb{Z}^{n}+2^{r-1} C_{r-1}\left(n, k_{r-1}\right)+\ldots \ldots+C_{0}\left(n, k_{0}\right)
$$

On the receiver side, a practical decoding algorithm is used, known as multistage decoding. The idea behind is that every code is decoded individually, in other words, we decode $C_{0}$, and then based on the estimated codeword we decode $C_{1}$ and so forth, until we have decoded all the codewords. To decode code $C_{i}$, we implement a soft-decision decoding algorithm, therefore, at each stage we need to calculate a soft-input which is the Log-Likelihood Ratio. However, LLR computations induce infinite sums of exponentials. It is then time and energy consuming [7]. In this paper, we deal with several lattice constructions each corrresponding to different lattice partition chain. The choice of such construction is related to ideals and their factorization; Hereinafter, we consider fields of characteristic 2 . For example $\mathbb{F}_{2}, \mathbb{F}_{4}, \mathbb{F}_{8}$. We also consider lattices based on complex constellations $\mathbb{Z}[\beta]$ where $\beta$ is a complex root of a quadratic polynomial with coefficients in $\mathbb{Z}$.

The choice of the construction is based on the prime factorization of the principal ideal generated by 2 ; we have three different cases:

- 2 ramifies in $\mathbb{Z}[\beta]$,so we have $2 \mathbb{Z}[\beta]=I^{2}$, where $I$ is the prime ideal: Binary construction $D$

- 2 remains inert over $\mathbb{Z}[\beta]$, so we have $2 \mathbb{Z}[\beta]$ is a prime over $\mathbb{Z}[\beta]$ : Quaternary construction $D$

- 2 splits into two distinct prime ideals over $\mathbb{Z}[\beta]$, so we have $2 \mathbb{Z}[\beta]=I \cdot \bar{I}$ : Binary construction $\pi_{D}$

For each of these three cases, we consider one example:

- 2 ramifies: $\beta=i$

- 2 is inert: $\beta=\omega=\frac{-1+i \sqrt{3}}{2}$

- 2 splits: $\beta=\frac{1+\sqrt{-7}}{2}$

The prime ideals resulting from the factorization of 2 have a lattice structure and are in fact sublattices of $\Lambda_{1}$, let's call them $\Lambda_{2}$

1) System Model: The $n$-dimensional lattice point $\mathbf{x}$ is sent through an AWGN channel giving the received point $\mathbf{y}=\mathbf{x}+\mathbf{w}$, where $\mathbf{w}$ is a length-n vector of zero-mean $\sigma^{2}$ variance gaussian distributed independant random variables. We consider a constellation carved out from a lattice $\Lambda_{1}$ and we consider the lattice partition $\Lambda_{1} / \Lambda_{2}$. A multistage decoding algorithm was introduced in [8] and [9] which feeds soft values of the received observation $\mathbf{y}$ into the binary decoder at each level. Each level of the construction $D$ may be viewed as construction $A$. We consider the previous example (2) and the received signal $\mathbf{y}=\mathbf{x}+\mathbf{w}$ so MSD can be described as follows:

- First $\mathbf{y}$ is reduced to $\mathbf{y}_{1}^{\prime} \equiv \mathbf{y} \bmod 2$, only the codeword corresponding to the code of the smallest rate remains.

- The soft-inputs to the decoder are then calculated based on the value of $\mathbf{y}_{1}^{\prime}$. Based on these values, the decoder of the code $C_{0}$ finds a decoded codeword $\hat{c}_{0}$. The estimated codeword is then substracted from $\mathbf{y}$ to give $\tilde{\mathbf{y}}=\mathbf{y}-\hat{c_{0}}$

- The next step will be to divide $\tilde{\mathbf{y}}$ by 2 , the modulo operation is again performed to get $\tilde{\mathbf{y}}_{2}^{\prime}$ and the LLRs are calculated based on the values of $\tilde{\mathbf{y}}_{2}^{\prime}$

- All the steps listed above are repeated until we get all the estimated codewords that correspond to the nested codes $C_{i}$

- Finally, only the element of $\mathbb{Z}^{n}$ that we call $b$ remains. Then a decoder for $\mathbb{Z}^{n}$ finds a sequence $\hat{\mathbf{b}}$ and we get the estimated lattice point $\hat{\mathbf{y}}$

So as we can see, due to the structure of the multistage decoder, the received signal is, at each level, subject to a modulo 2 operation. The resulting signal can be written as follows:

$$
\mathbf{y}^{\prime}=\mathbf{y} \quad \bmod 2=\mathbf{x} \quad \bmod 2+\mathbf{w} \quad \bmod 2=c_{0}+\mathbf{w}^{\prime}
$$

It follows from (3) that $c_{0} \in C_{0}$. The modulo 2 operation folds up the Gaussian Noise resulting in a vector $\mathbf{w}^{\prime}$ distributed according to the Wrapped Normal distribution [10]. Its probability density function (PDF) $f_{\sigma^{2}}\left(w^{\prime}\right)$. Let $g_{\sigma^{2}}(w)$ be the probability density function of the Gaussian noise $w \in \mathbb{R}^{n}$. $g_{\sigma^{2}}(w)$ and $f_{\sigma^{2}}\left(w^{\prime}\right)$ can be written as follows:

$$
\begin{aligned}
& g_{\sigma^{2}}(w)=\left(2 \pi \sigma^{2}\right)^{\frac{-n}{2}} e^{\frac{-\|w\|^{2}}{2 \sigma^{2}}} \\
& f_{\sigma^{2}}\left(w^{\prime}\right)=\sum_{b \in 2 \mathbb{Z}^{n}} g_{\sigma^{2}}\left(w^{\prime}+b\right)
\end{aligned}
$$

$w^{\prime}$ lives in the fundamental Voronoi region of $2 \mathbb{Z}^{n}$ that we call $R_{V}\left(2 \mathbb{Z}^{n}\right)$.

To decode $c_{i}$ we use a soft-decision decoder, consequently we must at each stage calculate the decoder soft-inputs which are Log-Likelihood Ratios. The caculations are very complex especially when we have several levels. Thus to simplify the multistage decoding algorithm, we propose an efficient computation of LLRs based only on Jacobi theta functions. In the non-binary case LLR vectors will be defined.

2) Jacobi Theta Functions: To each lattice $\Lambda$ we can associate a Jacobi theta function $\Theta_{\Lambda}$. Jacobi theta functions are the elliptic analogues of the exponential functions. There are several closely related functions called Jacobi theta functions, and many different and incompatible systems of notation for them. One Jacobi theta function defined as a function of two variables $\mathbf{u}$ and $\tau$, where $\mathbf{u}$ is a complex number and $\tau$ is defined in the upper half plane. 


$$
\Theta_{\Lambda}(\mathbf{u}, q)=\sum_{n=-\infty}^{\infty} q^{n^{2}} e^{2 i n \mathbf{u}}
$$

where $q=e^{i \pi \tau}$. There are four different types of elementary Jacobi theta functions. Hereinafter, we will be interested in $\vartheta_{2}$ and $\vartheta_{3}$. Note that these two types are even functions of the variable $\mathbf{u}$

$$
\begin{gathered}
\vartheta_{3}(\mathbf{u}, q)=\sum_{k=-\infty}^{\infty} q^{k^{2}} e^{2 i k \mathbf{u}} \\
\vartheta_{2}(\mathbf{u}, q)=\sum_{k=-\infty}^{\infty} q^{\left(k+\frac{1}{2}\right)^{2}} e^{(2 k+1) i \mathbf{u}}
\end{gathered}
$$

3) Computation rules of theta functions: Here we introduce some fundamental Jacobi theta functions'computation rules that we will exploit later for the calculation of the different LLRs.

- Scaling: $\Theta_{\alpha \Lambda_{1}}(\mathbf{u}, q)=\Theta_{\Lambda_{1}}\left(\alpha \mathbf{u}, q^{\alpha^{2}}\right) ; \alpha>0$

- Direct Sum: $\Theta_{\Lambda_{1} \oplus \Lambda_{2}}(\mathbf{u}, q)=\Theta_{\Lambda_{1}}\left(u_{1}, q\right) \Theta_{\Lambda_{2}}\left(u_{2}, q\right)$ $\mathbf{u}=\left(u_{1}, u_{2}\right)$

- Union: $\Theta_{\Lambda_{1} \cup \Lambda_{2}}(\mathbf{u}, q)=\Theta_{\Lambda_{1}}(\mathbf{u}, q)+\Theta_{\Lambda_{2}}(\mathbf{u}, q)$

\section{EFFICIENT LLR COMPUTATION BASED ON JACOBI THETA FUNCTIONS FOR GAUSSIAN CHANNELS}

\section{A. Binary Construction D}

1) Real Case: In this section, we consider the onedimensional partition chain $\mathbb{Z} / 2 \mathbb{Z} / 4 \mathbb{Z} \ldots \ldots / 2^{r} \mathbb{Z}$. As we can see, the quotient ring $\mathbb{Z} / 2 \mathbb{Z}$ has index 2 , and $\mathbb{Z}$ is the disjoint union of two cosets $2 \mathbb{Z}$ and $2 \mathbb{Z}+1$. We define the set of coset representatives as $A=\{0,1\}$. Here, Log-Likelihood Ratios are defined as follows:

$$
\mathrm{LLR}=\log \frac{\mathbb{P}\left(y_{i j}^{\prime} \mid x_{i j}=0\right)}{\mathbb{P}\left(y_{i j}^{\prime} \mid x_{i j}=1\right)}
$$

The theta functions of $\mathbb{Z}$ and $\mathbb{Z}+\frac{1}{2}$ can be written in terms of $\vartheta_{3}$ and $\vartheta_{2}$. Known that $\vartheta_{2}$ can also be written in terms of $\vartheta_{3}$, all the given results can be reduced to functions of $\vartheta_{3}$.

$$
\begin{aligned}
\Theta_{\mathbb{Z}}\left(\mathbf{y}, \sigma^{2}\right) & =\sum_{x \in \mathbb{Z}} e^{2 i \frac{i \mathbf{y}}{2 \sigma^{2}} x} e^{\frac{-x^{2}}{2 \sigma^{2}}} \\
& =\vartheta_{3}\left(\frac{i \mathbf{y}}{2 \sigma^{2}}, e^{\frac{-1}{2 \sigma^{2}}}\right) \\
\Theta_{\mathbb{Z}+\frac{1}{2}}\left(\mathbf{y}, \sigma^{2}\right) & =\sum_{x \in \mathbb{Z}} e^{2 i \frac{i \mathbf{y}}{2 \sigma^{2}}(2 x+1)} e^{\frac{-\left(x+\frac{1}{2}\right)^{2}}{2 \sigma^{2}}} \\
& =\vartheta_{2}\left(\frac{i \mathbf{y}}{2 \sigma^{2}}, e^{\frac{-1}{2 \sigma^{2}}}\right)
\end{aligned}
$$

Now, using the computation rules given above, we have the following results for the cosets $2 \mathbb{Z}$ and $2 \mathbb{Z}+1$ :

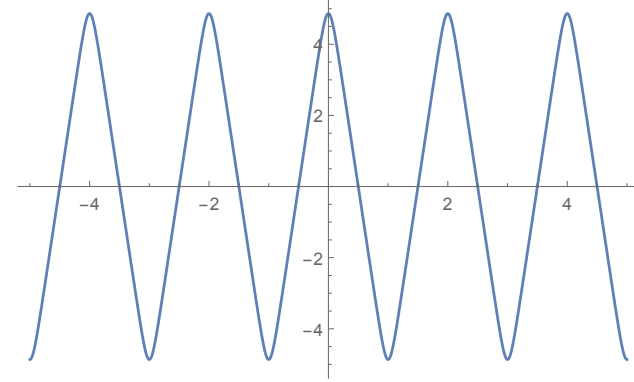

Fig. 1. LLRs for the binary real construction $D$ for $\sigma=0.2 \mathrm{db}$

$$
\begin{gathered}
\Theta_{2 \mathbb{Z}}\left(\mathbf{y}, \sigma^{2}\right)=\sum_{x \in \mathbb{Z}} e^{2 i \frac{i \mathbf{y}}{\sigma^{2}} x} e^{\frac{-2 x^{2}}{\sigma^{2}}} \\
\Theta_{2 \mathbb{Z}+1}\left(\mathbf{y}, \sigma^{2}\right)=\sum_{x \in \mathbb{Z}} e^{2 i \frac{i \mathbf{y}}{\sigma^{2}}(2 x+1)} e^{\frac{-2\left(x+\frac{1}{2}\right)^{2}}{\sigma^{2}}}
\end{gathered}
$$

Then Log-Likelihood Ratios can be defined as follows:

$$
\begin{aligned}
\mathbb{P}(y \mid x=0) & =\frac{1}{\sqrt{2 \pi} \sigma} \sum_{k \in \mathbb{Z}} e^{\frac{-\|y-2 k\|^{2}}{2 \sigma^{2}}} \\
& =\frac{e^{\frac{-\|y\|^{2}}{2 \sigma^{2}}}}{\sqrt{2 \pi} \sigma} \sum_{k \in \mathbb{Z}} e^{\frac{2 y k}{\sigma^{2}}} e^{\frac{-2 k^{2}}{\sigma^{2}}} \\
& =\frac{e^{\frac{-\|y\|^{2}}{2 \sigma^{2}}}}{\sqrt{2 \pi} \sigma} \vartheta_{3}\left(\frac{i y}{\sigma^{2}}, e^{\frac{-2}{\sigma^{2}}}\right) \\
\mathbb{P}(y \mid x=1)= & \sum_{k \in \mathbb{Z}} e^{\frac{-\|y-(2 k+1)\|^{2}}{2 \sigma^{2}}} \\
= & \frac{e^{\frac{-\|y\|^{2}}{2 \sigma^{2}}}}{\sqrt{2 \pi} \sigma} \sum_{k \in \mathbb{Z}} e^{\frac{y(2 k+1)}{\sigma^{2}}} e^{\frac{-2\left(k+\frac{1}{2}\right)^{2}}{\sigma^{2}}} \\
= & \frac{e^{\frac{-\|y\|^{2}}{2 \sigma^{2}}}}{\sqrt{2 \pi} \sigma} \vartheta_{2}\left(\frac{i y}{\sigma^{2}}, e^{\frac{-2}{\sigma^{2}}}\right) \\
\operatorname{LLR} & =\log \frac{\mathbb{P}(y \mid x=0)}{\mathbb{P}(y \mid x=1)} \\
& =\log \frac{\vartheta_{3}\left(\frac{i y}{\sigma^{2}}, e^{\frac{-2}{\sigma^{2}}}\right)}{\vartheta_{2}\left(\frac{i y}{\sigma^{2}}, e^{\frac{-2}{\sigma^{2}}}\right)}
\end{aligned}
$$

LLRs in the case of a real binary construction $D$ are shown in figure 1.

2) Complex Case: (2 ramifies): Complex binary construction $D$ is also another way to construct lattices. In this case, we consider the two-dimensional lattice partition chain $\mathbb{Z}[i] /(1+i) \mathbb{Z}[i] / 2 \mathbb{Z}[i] \ldots$, where $\mathbb{Z}[i]$ is the ring of Gaussian integers. As in the real case the set of coset representatives is defined this way $A=\{0,1\}$.

Known that, $\mathbb{Z}[i] /(1+i) \mathbb{Z}[i]$ is equivalent to $\mathbb{Z}^{2} / D_{2}$ where

$$
D_{2}=2 \mathbb{Z}^{2}+(2,1)_{\mathbb{F}_{2}}=(2 \mathbb{Z} \oplus 2 \mathbb{Z}) \cup((2 \mathbb{Z}+1) \oplus(2 \mathbb{Z}+1))
$$




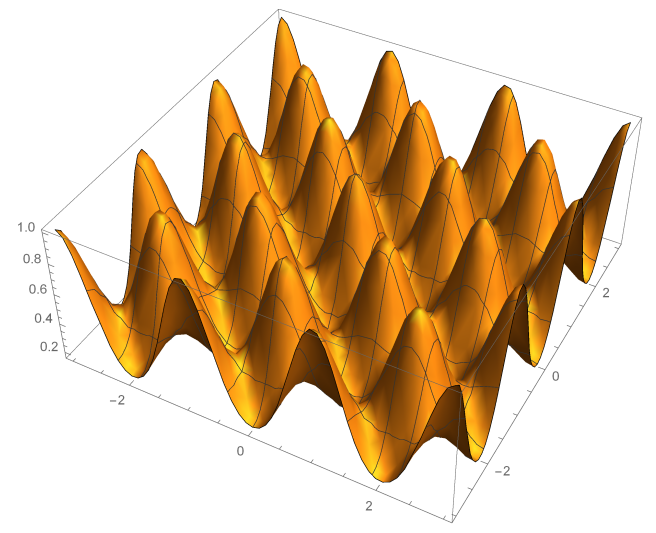

Fig. 2. A-posteriori probability of the first coset $D_{2}$ corresponding to $\sigma=$ $0.4 \mathrm{db}$

We can calculate $\Theta_{D_{2}}$ this way:

$$
\begin{aligned}
\Theta_{D_{2}}\left(\mathbf{y}, \sigma^{2}\right) & =\Theta_{2 \mathbb{Z}}\left(y_{1}, \sigma^{2}\right) \Theta_{2 \mathbb{Z}}\left(y_{2}, \sigma^{2}\right) \\
& +\Theta_{2 \mathbb{Z}+1}\left(y_{1}, \sigma^{2}\right) \Theta_{2 \mathbb{Z}+1}\left(y_{2}, \sigma^{2}\right) \\
& =\vartheta_{3}\left(\frac{i y_{1}}{\sigma^{2}}, e^{\frac{-2}{\sigma^{2}}}\right) \vartheta_{3}\left(\frac{i y_{2}}{\sigma^{2}}, e^{\frac{-2}{\sigma^{2}}}\right) \\
& +\vartheta_{2}\left(\frac{i y_{1}}{\sigma^{2}}, e^{\frac{-2}{\sigma^{2}}}\right) \vartheta_{2}\left(\frac{i y_{2}}{\sigma^{2}}, e^{\frac{-2}{\sigma^{2}}}\right)
\end{aligned}
$$

where $\mathbf{y}=\left(y_{1}, y_{2}\right)$.

The a posteriori probability can be written as follows:

$$
\begin{aligned}
\mathbb{P}\left(\mathbf{y} \mid x \in D_{2}\right) & =\frac{e^{\frac{-\left(y_{1}^{2}+y_{2}^{2}\right)}{2 \sigma^{2}}}}{2 \pi \sigma}\left(\vartheta_{3}\left(\frac{i y_{1}}{\sigma^{2}}, e^{\frac{-2}{\sigma^{2}}}\right) \vartheta_{3}\left(\frac{i y_{2}}{\sigma^{2}}, e^{\frac{-2}{\sigma^{2}}}\right)\right. \\
& \left.+\vartheta_{2}\left(\frac{i y_{1}}{\sigma^{2}}, e^{\frac{-2}{\sigma^{2}}}\right) \vartheta_{2}\left(\frac{i y_{2}}{\sigma^{2}}, e^{\frac{-2}{\sigma^{2}}}\right)\right)
\end{aligned}
$$

An illustration of the a posteriori probability of $D_{2}$ is given in figure 2.

The second coset is a simple translation of the first one. Then LLRs have this expression:

$$
\mathrm{LLR}=\log \frac{\mathbb{P}\left(y \mid x \in D_{2}\right)}{\mathbb{P}\left(y \mid D_{2}+(1,0)_{\mathbb{F}_{2}}\right)}
$$

\section{B. Quaternary Construction D}

(2 is inert): By choosing a bigger alphabet size, one can increase coding gain. Quaternary contruction $D$ is based on partition chains of the densest lattice in dimension 2 which is the hexagonal lattice $A_{2}$. Here we consider the twodimensional, four-way partition $\mathbb{Z}[\omega] / 2 \mathbb{Z}[\omega] / 4 \mathbb{Z}[\omega] .$. , where $\omega=e^{\frac{2 i \pi}{3}} . \mathbb{Z}[\omega]$ is called the ring of the Eisenstein integers. Log-Likelihood Ratios can be defined as length-three vectors normalized with respect to $\mathbb{P}(y \mid x=0)$ :

$$
\text { LLR }=\left(\begin{array}{c}
\log \frac{\mathbb{P}(y \mid x=1)}{\mathbb{P}(y \mid x=0)} \\
\log \frac{\mathbb{P}(y \mid x=\alpha)}{\mathbb{P}(y \mid x=0)} \\
\log \frac{\mathbb{P}\left(y \mid x=\alpha^{2}\right)}{\mathbb{P}(y \mid x=0)}
\end{array}\right)
$$

Here, the partition index is $|\mathbb{Z}[\omega] / 2 \mathbb{Z}[\omega]|=4$ and the quotient ring $\mathbb{Z}[\omega] / 2 \mathbb{Z}[\omega]$ is isomomorphic to $\mathbb{F}_{4}$ (see Table I)

We consider $\mathbb{Z}[\omega] / 2 \mathbb{Z}[\omega]$ which is equivalent to $\mathbb{Z}^{2} / 2 A_{2}$.
TABLE I

Coset RePresentatives of ELEMENTS of $\mathbb{F}_{4}$

\begin{tabular}{|c||c|}
\hline $\mathbb{Z}[\omega]$ & $\mathbb{F}_{4}$ \\
\hline \hline 0 & 0 \\
\hline 1 & 1 \\
\hline$\omega$ & $\alpha$ \\
\hline$\omega^{2}$ & $\alpha^{2}$ \\
\hline
\end{tabular}

$$
A_{2}=(\mathbb{Z} \oplus \sqrt{3} \mathbb{Z}) \cup\left(\left(\mathbb{Z}+\frac{1}{2}\right) \oplus\left(\mathbb{Z}+\frac{1}{2}\right) \sqrt{3}\right)
$$

Then:

$\Theta_{A_{2}}(\mathbf{u}, q)=\vartheta_{3}\left(u_{1}, q\right) \vartheta_{3}\left(\sqrt{3} u_{2}, q^{3}\right)+\vartheta_{2}\left(u_{1}, q\right) \vartheta_{2}\left(\sqrt{3} u_{2}, q^{3}\right)$

And

$$
\begin{aligned}
\Theta_{2 A_{2}}(\mathbf{u}, q) & =\vartheta_{3}\left(2 u_{1}, q^{4}\right) \vartheta_{3}\left(2 \sqrt{3} u_{2}, q^{12}\right) \\
& +\vartheta_{2}\left(2 u_{1}, q^{4}\right) \vartheta_{2}\left(2 \sqrt{3} u_{2}, q^{12}\right)
\end{aligned}
$$

Now, known that cosets $2 A_{2}+\omega$ and $2 A_{2}+\bar{\omega}$ have the same Jacobi theta function:

$$
\begin{aligned}
\Theta_{2 A_{2}+1}(\mathbf{u}, q) & =\vartheta_{3}\left(u_{1}, q\right) \vartheta_{2}\left(\sqrt{3} u_{2}, q^{3}\right) \\
& +\vartheta_{2}\left(u_{1}, q\right) \vartheta_{3}\left(\sqrt{3} u_{2}, q^{3}\right)
\end{aligned}
$$

Where the variables $\mathbf{u}=\left(u_{1}, u_{2}\right)$ such that $u_{1}=\frac{i y_{1}}{\sigma^{2}}, u_{2}=\frac{i y_{2}}{\sigma^{2}}$ and $q=e^{\frac{-1}{2 \sigma^{2}}}$ as they were defined for the theta functions associated to $\mathbb{Z}$ and $\mathbb{Z}+\frac{1}{2}$. Then:

$$
\begin{aligned}
\Theta_{2 A_{2}+\omega}(\mathbf{u}, q) & =\Theta_{2 A_{2}+\bar{\omega}}(\mathbf{u}, q) \\
& =\frac{\Theta_{A_{2}}(\mathbf{u}, q)-\Theta_{2 A_{2}}(\mathbf{u}, q)-\Theta_{2 A_{2}+1}(\mathbf{u}, q)}{2}
\end{aligned}
$$

The a posteriori probability expression is then equal to:

$$
\mathbb{P}(y \mid x=\lambda)=\frac{1}{2 \pi \sigma} e^{\frac{\|y\|^{2}}{2 \sigma^{2}}} \Theta_{2 A_{2}+\lambda}\left(\frac{i y}{2 \sigma^{2}}, e^{\frac{-1}{2 \sigma^{2}}}\right)
$$

where $\lambda \in\{0,1, \omega, \bar{\omega}\}$

\section{Binary construction $\pi_{D}$}

(2 splits): Construction $\pi_{D}$ is a generalization of all constructions, it subsumes construction $A$, construction $D$ and construction $\pi_{A}$. It has been introduced in [11]. As for construction $D$, this construction is based on multivel coding and multistage decoding thus LLR calculations are needed at each stage. The decoding algorithm is very close to the decoding algorithm of construction $D$, however, in this case we have two lattice partition chains; $\mathbb{Z}[\alpha] / I / 2 \mathbb{Z}[\alpha]$ and $\mathbb{Z}[\alpha] / \bar{I} / 2 \mathbb{Z}[\alpha]$, where $\alpha=\frac{1+\sqrt{-7}}{2}$ and we have a family of nested equivalent codes. Coset representatives will be here couples $\left(a_{1}, a_{2}\right)$ in $\mathbb{F}_{2} \times \mathbb{F}_{2}$ (see table II).

$\mathbb{Z}[\alpha]$ can be written as the product of an ideal $I$ and its conjugate $\bar{I}, 2 \mathbb{Z}[\alpha]=I \bar{I}$

The lattice $\mathbb{Z}[\alpha]$ can be defined as follows:

$$
\mathbb{Z}[\alpha]=(\mathbb{Z} \oplus \sqrt{7} \mathbb{Z}) \cup\left(\left(\mathbb{Z}+\frac{1}{2}\right) \oplus \sqrt{7}\left(\mathbb{Z}+\frac{1}{2}\right)\right)
$$




$$
\operatorname{LLR}=\log \left[\frac{\vartheta_{3}\left(\frac{i y_{1}}{\sigma^{2}}, e^{\frac{-2}{\sigma^{2}}}\right) \vartheta_{3}\left(\frac{i y_{2}}{\sigma^{2}}, e^{\frac{-2}{\sigma^{2}}}\right)+\vartheta_{2}\left(\frac{i y_{1}}{\sigma^{2}}, e^{\frac{-2}{\sigma^{2}}}\right) \vartheta_{2}\left(\frac{i y_{2}}{\sigma^{2}}, e^{\frac{-2}{\sigma^{2}}}\right)}{\vartheta_{3}\left(\frac{i y_{1}}{\sigma^{2}}, e^{\frac{-2}{\sigma^{2}}}\right) \vartheta_{2}\left(\frac{i y_{2}}{\sigma^{2}}, e^{\frac{-2}{\sigma^{2}}}\right)+\vartheta_{2}\left(\frac{i y_{1}}{\sigma^{2}}, e^{\frac{-2}{\sigma^{2}}}\right) \vartheta_{3}\left(\frac{i y_{2}}{\sigma^{2}}, e^{\frac{-2}{\sigma^{2}}}\right)}\right]
$$

TABLE II

Coset Representatives of ELEMENTS of $\mathbb{F}_{2} \times \mathbb{F}_{2}$

\begin{tabular}{|c||c|}
\hline $\mathbb{Z}[\alpha]$ & $\mathbb{F}_{2} \times \mathbb{F}_{2}$ \\
\hline \hline 0 & $(0,0)$ \\
\hline 1 & $(1,1)$ \\
\hline$\alpha$ & $(0,1)$ \\
\hline $\bar{\alpha}$ & $(1,0)$ \\
\hline
\end{tabular}

The Jacobi theta function associated to this lattice is:

$$
\begin{aligned}
\Theta_{\mathbb{Z}[\alpha]}\left(\mathbf{y}, \sigma^{2}\right) & =\vartheta_{3}\left(\frac{i y_{1}}{2 \sigma^{2}}, e^{\frac{-1}{2 \sigma^{2}}}\right) \vartheta_{3}\left(\frac{\sqrt{7} i y_{2}}{2 \sigma^{2}}, e^{\frac{-7}{2 \sigma^{2}}}\right) \\
& +\vartheta_{2}\left(\frac{i y_{1}}{2 \sigma^{2}}, e^{\frac{-1}{2 \sigma^{2}}}\right) \vartheta_{2}\left(\frac{\sqrt{7} i y_{2}}{2 \sigma^{2}}, e^{\frac{-7}{2 \sigma^{2}}}\right)
\end{aligned}
$$

where $\mathbf{y}=\left(y_{1}, y_{2}\right)$. Now, $\mathbb{Z}[\alpha]$ can be written as the union of four disjoint cosets:

$$
\mathbb{Z}[\alpha]=2 \mathbb{Z}[\alpha] \cup(2 \mathbb{Z}[\alpha]+1) \cup(2 \mathbb{Z}[\alpha]+\alpha) \cup(2 \mathbb{Z}[\alpha]+\bar{\alpha})
$$

$(2 \mathbb{Z}[\alpha]+\alpha)$ and $(2 \mathbb{Z}[\alpha]+\bar{\alpha})$ have the same theta function:

$\Theta_{\mathbb{Z}[\alpha]}(\mathbf{u}, q)=\Theta_{2 \mathbb{Z}[\alpha]}(\mathbf{u}, q)+\Theta_{2 \mathbb{Z}[\alpha]+1}(\mathbf{u}, q)+2 \Theta_{2 \mathbb{Z}[\alpha]+\alpha}(\mathbf{u}, q)$

On the other hand, we have:

$$
\begin{aligned}
\Theta_{2 \mathbb{Z}[\alpha]}\left(\mathbf{y}, \sigma^{2}\right) & =\vartheta_{3}\left(\frac{2 i y_{1}}{2 \sigma^{2}}, e^{\frac{-2}{\sigma^{2}}}\right) \vartheta_{3}\left(\frac{2 \sqrt{7} i y_{2}}{2 \sigma^{2}}, e^{\frac{-14}{\sigma^{2}}}\right) \\
& +\vartheta_{2}\left(\frac{2 i y_{1}}{2 \sigma^{2}}, e^{\frac{-2}{\sigma^{2}}}\right) \vartheta_{2}\left(\frac{2 \sqrt{7} i y_{2}}{2 \sigma^{2}}, e^{\frac{-14}{\sigma^{2}}}\right) \\
\Theta_{2 \mathbb{Z}[\alpha]+1}\left(\mathbf{y}, \sigma^{2}\right) & =\vartheta_{3}\left(\frac{2 i y_{1}}{2 \sigma^{2}}, e^{\frac{-2}{\sigma^{2}}}\right) \vartheta_{2}\left(\frac{2 \sqrt{7} i y_{2}}{2 \sigma^{2}}, e^{\frac{-14}{\sigma^{2}}}\right) \\
& +\vartheta_{2}\left(\frac{2 i y_{1}}{2 \sigma^{2}}, e^{\frac{-2}{\sigma^{2}}}\right) \vartheta_{3}\left(\frac{2 \sqrt{7} i y_{2}}{2 \sigma^{2}}, e^{\frac{-14}{\sigma^{2}}}\right)
\end{aligned}
$$

where $\mathbf{y}=\left(y_{1}, y_{2}\right)$. Then:

$$
\begin{aligned}
\Theta_{2 \mathbb{Z}[\alpha]+\alpha}(\mathbf{u}, q) & =\Theta_{2 \mathbb{Z}[\alpha]+\bar{\alpha}}(\mathbf{u}, q) \\
& =\frac{\Theta_{\mathbb{Z}[\alpha]}(\mathbf{u}, q)-\Theta_{2 \mathbb{Z}[\alpha]}(\mathbf{u}, q)-\Theta_{2 \mathbb{Z}[\alpha]+1}(\mathbf{u}, q)}{2}
\end{aligned}
$$

\section{CONCLUSION}

We showed in this paper that the knowledge of the value of two types of Jacobi theta functions $\vartheta_{3}$ and $\vartheta_{2}$ is sufficient for computing LLRs when constructions $D$ or $\pi_{D}$ are used to construct high dimensional lattice codes. Known that $\vartheta_{2}$ can be expressed in terms of $\vartheta_{3}$, the expressions of LLRs can even be simpler. The calculation of Log-Likelihhod Ratios is then simplified; instead of having to calculate infinite sums of exponentials, one can have simple look-up tables containing values of Jacobi theta function $\vartheta_{3}$.

\section{REFERENCES}

[1] S Zhang SC Liew and P P Lam, Hot-topic Physical layer network coding. in Proc, 12th MobiCom, pages 358-365, New York, NY, USA 2006.

[2] B. Nazer and M. Gastpar. Compute-and-forward: Harnessing interference through structured codes. Information theory,IEEE transactions, vol.57,pp.6463-6486 Oct2011.

[3] J. Conway and N.J..A Sloane, sphere Packings, Lattices and groups. Springer New York,2010

[4] H. Imai and S. Hirakawa A New multiLevel coding method using errorcorrecting codes. Information Theory, IEEE Transactionson, vol.23,pp 371-377, May 1977

[5] G. Forney, M. Trott, and S.,-Y. Chung Sphere-bound achieving coset codes and multilevel coset codes. Information Theory, IEEE Transactionson, vol.46,pp 820-850, May 2000

[6] G.D. Forney Jr., A bounded-distance decoding algorithm for the leech lattice, with generalizations. IEEE trans. Inf. Theory,vol.35,pp 906-909, July 1989

[7] Guido Montorsi and Farbod Kayhan Analog Digital Belief Propagation and its application to multi stage decoding systems. In Communications and Networking (BlackSeaCom), 2015 IEEE International Black Sea Conference on (pp. 82-86). IEEE May 2015

[8] A. Sakzad, M.-R. Sadeghi and D. Panario Construction of turbo lattices. In Allerton Conference on Communication,Control, and computing, 2010,Allerton,IL, pp. 14-21 Sept29-Oct1 2010

[9] K. Mardia, Statistics of Directional Data. Academic Press, 1972

[10] Yuh-Chih Huang and Krishna R. Narayanan Construction $\pi_{A}$ and $\pi_{D}$ Lattices: Construction, Goodness, and decoding algorithms. International Symposium on Information Theory 2014 